\title{
Gestión de cuentas por cobrar y la rentabilidad de las empresas del sector industrial que cotizan en la Bolsa de Valores de Lima, períodos 2010 al 2014
}

\author{
Mitzy Katherine Flores Morales ${ }^{12 a}$ y Yanina Naval Carrasco ${ }^{12}$
}

Universidad Peruana Unión, Perú12

Nestlé Perú S. A ${ }^{12}$

Recibido: 12 de enero de 2016

Aceptado: 13 de mayo de 2016

\section{Resumen}

El objetivo de este trabajo es identificar la relación entre gestión de cuentas por cobrar y la rentabilidad de las empresas del sector industrial que cotizan en la Bolsa de Valores de Lima, períodos 2010 al 2014. El trabajo es de diseño no experimental de tipo análisis de descriptivo - correlacional. Es de corte transversal. Es retrospectivo ya que se toma a las empresas industriales que cotizan en la Bolsa de Valores de Lima en los periodos 2010 - 2014. Los indicadores que intervienen en el cálculo son las ventas anuales, las cuentas por cobrar, el costo de venta, las cuentas por pagar, inventario. Los resultados expresan que existe correlación entre los indicadores de gestión de actividad y la rentabilidad económica de las empresas del sector industrial que cotizan en BVL 2010 al 2014. Mediante el análisis estadístico R de Pearson se obtuvo que los indicadores rotación de cuentas por cobrar (veces), periodo promedio de cobranza (días) y rotación de inventarios son altamente significativos, por lo tanto, se rechaza la hipótesis nula y se acepta la hipótesis alterna, es decir que los indicadores rotación de cuentas por cobrar (veces), periodo promedio de cobranza (días) y rotación de inventarios se relacionan favorablemente con la rentabilidad económica.

Palabras clave: Gestión de actividad, rentabilidad, capital de trabajo, bolsa de valores

\section{Relationship of the management of accounts receivable and the profitability of the companies of the industrial sector that are listed on the Lima Stock Exchange, periods 2010 to 2014}

\begin{abstract}
The main of this paper is to identify the relationship between management of accounts receivable and the profitability of companies in the industrial sector that are listed on the Lima Stock Exchange, from 2010 to 2014. The work has a non-experimental design and uses correlational descriptive analysis. It is based on a cross section and it is retrospective since it takes industrial companies listed on the Lima Stock Exchange
\end{abstract}


(BVL) in the periods 2010 - 2014. The indicators involved in the calculation are annual sales, accounts receivable, cost of sale, accounts payable and inventory. The results show that there is a correlation between the indicators of activity management and the economic profitability of the companies of the industrial sector that are listed in BVL 2010 to 2014. By means of the Pearson's R statistical analysis, it was pbserved that the indicators of accounts receivable rotation (times), average collection period (days) and inventory rotation are highly significant, therefore, the null hypothesis is rejected and the alternative hypothesis is accepted, that is, the indicators of accounts receivable rotation (times), average period of collection (days) and inventory turnover are favorably related to economic profitability.

Keywords: Activity management, profitability, working capital, accounts receivable, accounts payable, inventory rotation.

\section{Introducción}

La teoría sobre el capital se inició con los supuestos de Smith (1794) sosteniendo que la riqueza era fruto del trabajo de la propiedad privada que se adquiría a raíz de un capital que se había invertido y generado beneficios. Locke (2010) consideró el capital como propiedad privada que tenía una persona, esto lo que podía obtener o desarrollar con su trabajo como beneficio monetario a cambio de esta. Posteriormente, De Mariana (1854) alude los inconvenientes de esta teoría, indicando que el tirano tomaba por suyo los bienes y la propiedad privada de sus vasallos para enriquecerse.

En los últimos años el término de capital de trabajo pasó de ser solo un cálculo. Espinoza \& Selpa (2004) comienzan a definir la gestión del capital de trabajo como un proceso. Aching \& Aching (2006) comienzan a medir la efectividad de la gestión de actividad en la administración del capital de trabajo.

Es así que Jamalinesari \& Soheili (2015) nos dicen que para tener una eficiente gestión de capital de trabajo es necesario tener una buena gestión de activos y pasivos. Pandey \& Sabamaithily (2016) centran su estudio en el impacto del capital de trabajo en la rentabilidad que afectan a la gestión del capital de trabajo. Akram, Jamil, Ali, Ali, \& Khan (2016) mencionan en que la gestión del capital es una excelente herramienta utilizada para gestionar la disposición de sus activos pasivos y corto plazo a corto plazo.

La gestión de actividad mide la efectividad de la correcta administración del capital de trabajo Aching \& Aching (2006). Es así que Jamalinesari \& Soheili (2015) nos dicen que para tener una eficiente gestión de capital de trabajo es necesario tener una buena gestión de activos y pasivos, Akram, Jamil, Ali, Ali, \& Khan (2016) mencionan en que la gestión del capital es una excelente herramienta utilizada para gestionar la disposición de sus activos pasivos y corto plazo a corto plazo. Espinoza \& Selpa (2004) se refiere al capital de trabajo como el excedente del activo circulante sobre el pasivo circulante y entre los autores que respaldan esto se encuentran: L. Gitman (1986); J. Tracy (1993); F. Weston y E. Brigham (1994); Maighs (1995); L. Bernstein (1997); R. Brealey (1998); O. Amat (1998); A. Demestre et al. (2002); A. Blanco (2004); G. Gómez (2004); R. Arévalo (2004) y F. Munilla et al. (2005).

Definen Perez-Carballo \& Perez-Carballo (1998); Pérez-Carballo (2013); Otal, Sara, Serrano, \& Serrano (2007); Saucedo \& Oyola (2014) a la rentabilidad económica, como medida de rendimiento del activo neto de 
la empresa, asimismo Moreno (2014) menciona que Pares (1979) opina que la rentabilidad económica o de la inversión, es una medida referida a un determinado periodo de tiempo, del rendimiento de los activos de una empresa con independencia de la financiación de los mismos.

En la actualidad la Bolsa de Valores de Lima cuenta con 44 empresas inscritas en el sector industrial. Según la revista América Economía al termino del 2013 la empresa industrial que destacaron fueron British American Tobacco con un ROE de 196,5\%.

Se tiene como referencia investigaciones realizadas por otros investigadores tal como el trabajo de investigación realizado Pandey \& Sabamaithily (2016) en su trabajo titulado "El capital de trabajo en la Rentabilidad: Industria del cemento en la India" tiene como objetivos analizar el impacto de la gestión del circulante en la rentabilidad, conocer los diversos factores que afectan a los requisitos de capital de trabajo en la industria del cemento, y examinar el crecimiento y el valor de la tendencia de la industria del cemento en la India durante el período del estudio. El estudio se basa en datos secundarios, donde se ha utilizado como base de datos la información recogida de moneycontrol.com, los estados financieros del estado de resultados y el de situación financiera de la industria del cemento seleccionadas. La unidad de muestra de este estudio ha sido elegida de la industria del cemento enumerado en la Bolsa de Bombay (BSE). Sólo se dispone de 24 industrias del cemento que aparece en la EEB, por tanto, 24 industrias han sido consideradas como tamaño de la muestra para este estudio. Y el período del estudio se ha considerado desde hace 10 años, es decir, 2004-05 a 2013-14. Como método de análisis se utilizó la Estadística Descriptiva, como la media y la desviación estándar, la tendencia central y la coherencia de los datos de series de tiempo. También se utilizó el análisis de regresión con la ayuda del paquete estadístico para Ciencias Sociales (SPSS). Tendiendo como conclusión, que va depender de la manera en que se gestiona el capital de trabajo tendrá un impacto significativo en la rentabilidad de estas empresas.

Singhania, Sharma, \& Yagnesh (2014) en su investigación "Gestión de Capital de trabajo y Rentabilidad: Evidencia de las empresas manufactureras de la India." El objetivo de este estudio fue analizar la relación entre las estrategias de gestión de capital de trabajo y la rentabilidad, para entender el impacto de las condiciones macroeconómicas globales específicas en el mismo. El alcance de este estudio de investigación se limita a las empresas manufactureras de la India que componen el índice BSE - 500 de la Bolsa de Bombay, las fuentes de los datos fueron los estados financieros consolidados de las empresas, los datos secundarios pertinentes de la base de datos de Capitalino de 2005-2012. Y la muestra final estuvo compuesta por un total de 656 observaciones empresa - año, que comprendía datos de 82 empresas de más de 8 años período. Se aplicó 4 modelos de análisis de regresión, haciendo uso del análisis de datos de panel, en particular, la estimación de efectos fijos, en nuestra muestra de datos. Se concluye que las decisiones en la gestión de capital tienen un impacto en la rentabilidad corporativa. Por lo tanto, el rendimiento de la empresa se puede mejorar mediante la adopción de estrategias de capital de trabajo adecuado.

El objetivo de este trabajo es identificar la relación entre gestión de cuentas por cobrar y la rentabilidad de las empresas del sector industrial que cotizan en la Bolsa de Valores de Lima, períodos 2010 al 2014. 


\section{Método}

En la presente investigación se tienen como variable predictora a la gestión de actividad cuyas dimensiones son gestión de cuentas por cobrar, gestión de cuentas por pagar y gestión de existencias. Variable criterio a la rentabilidad cuyas dimensiones son rentabilidad financiera, rentabilidad económica y rentabilidad sobre ventas. Para la presente investigación se plantearon las hipótesis: La gestión de actividad está relacionada con la rentabilidad económica de las empresas del sector industrial que cotizan en la Bolsa de Valores de Lima periodos 2010 al 2014 y la gestión de actividad está relacionada con la rentabilidad financiera de las empresas del sector industrial que cotizan en la Bolsa de Valores de Lima periodos 2010 al 2014.

Se tiene como objetivo general determinar la relación de la gestión actividad y la rentabilidad de las empresas del sector industrial que cotizan en la Bolsa de Valores de Lima periodos 2010 al 2014 y como objetivos específicos determinar la relación de la gestión de actividad y la rentabilidad económica de las empresas del sector industrial que cotizan en la Bolsa de Valores de Lima periodos 2010 al 2014, determinar la relación de la gestión de actividad y la rentabilidad financiera de las empresas del sector industrial que cotizan en la Bolsa de Valores de Lima periodos 2010 al 2014.

Se tomaron datos publicados en el portal web de la BLV correspondiente a las empresas del sector industrial que cotizan en la BVL los periodos 2010 al 2014. El presente trabajo de investigación solo incluye el sector industrial. En total son 44 empresas actualmente, sin embargo, realizada la limpieza de datos y aplicados los criterios de exclusión se analizan 38 empresas.

Se utilizó el programa estadístico informático SPSS donde se analizó el percentil 30 y percentil 70 para el nivel de la gestión de actividad y rentabilidad tales como bajo, medio, alto de acuerdo al sector industrial. Después se realizó un análisis de correlación a través del estadístico R de Pearson para hallar la relación que existe entre la gestión de actividad y rentabilidad.

\section{Resultados y discusión}

En la tabla 1 se muestra nivel promedio de la rotación de cuentas por cobrar (veces) de las empresas del sector industrial que cotizan en la BVL periodos 2010 al 2014. Entre los tres niveles se observa que el de mayor representación es el nivel bajo, y está comprendido por el $39.47 \%$ de las empresas, seguido de un 31.58 $\%$ que se ubican en un nivel medio y finalmente el $28.95 \%$ se ubican en un nivel alto de rotación de cuentas por cobrar (veces).

\section{Tabla 1}

Nivel promedio de la rotación de cuentas por cobrar (veces) de las empresas del sector industrial que cotizan en la BVL periodos 2010 al 2014

\begin{tabular}{lll}
\hline Nivel & Frecuencia & Porcentaje \\
\hline Bajo & 75 & 39.47 \\
Medio & 60 & 31.58 \\
Alto & 55 & 28.95
\end{tabular}


En la tabla 2 se muestra nivel del periodo promedio de cobranza (días) de las empresas del sector industrial que cotizan en la BVL periodos 2010 al 2014. Entre los tres niveles se observa que el de mayor representación es el nivel medio, y está comprendido por el 38.95\% de las empresas, seguido de un $31.05 \%$ que se ubican en un nivel bajo y finalmente el $30.00 \%$ de las empresas se ubican en un nivel alto de periodo promedio de cobranza (días).

Tabla 2

Nivel promedio del periodo promedio de cobranza (días) de las empresas del sector industrial que cotizan en la BVL periodos 2010 al 2014

\begin{tabular}{lll}
\hline Nivel & Frecuencia & Porcentaje \\
\hline Bajo & 59 & 31.05 \\
Medio & 74 & 38.95 \\
Alto & 57 & 30.00 \\
Total & 190 & 100.00 \\
\hline
\end{tabular}

En la tabla 3 se muestra nivel de la rotación de cuentas por pagar (veces) de las empresas del sector industrial que cotizan en la BVL periodos 2010 al 2014. Entre los tres niveles se observa que el de mayor representación es el nivel bajo, y está comprendido por el $42.63 \%$ de las empresas, seguido de un $28.95 \%$ que se ubican en un nivel alto y finalmente el $28.42 \%$ están comprendidos en un nivel medio.

Tabla 3

Nivel promedio de la rotación de cuentas por pagar (veces) de las empresas del sector industrial que cotizan en la BVL periodos 2010 al 2014

\begin{tabular}{lll}
\hline Nivel & Frecuencia & Porcentaje \\
\hline Bajo & 81 & 42.63 \\
Medio & 54 & 28.42 \\
Alto & 55 & 28.95 \\
Total & 190 & 100.00 \\
\hline
\end{tabular}

En la tabla 4 se muestra nivel de la rotación de cuentas de deuda (días) de las empresas del sector industrial que cotizan en la BVL periodos 2010 al 2014. Entre los tres niveles se observa que el de mayor representación es el nivel medio, y está comprendido por el $41.58 \%$ de las empresas, seguido de un $28.42 \%$ que se ubican en un nivel alto y finalmente el $30.00 \%$ están comprendidos en un nivel bajo.

Tabla 4

Nivel promedio de la rotación de cuentas de deuda (días) de las empresas del sector industrial que cotizan en la BVL periodos 2010 al 2014

\begin{tabular}{lll}
\hline Nivel & Frecuencia & Porcentaje \\
\hline Bajo & 57 & 30.00 \\
Medio & 79 & 41.58 \\
Alto & 54 & 28.42 \\
Total & 190 & 100.00 \\
\hline
\end{tabular}


En la tabla 5 se muestra nivel de la rotación de inventarios de las empresas del sector industrial que cotizan en la BVL periodos 2010 al 2014. Entre los tres niveles se observa que el de mayor representación es el nivel bajo, y está comprendido por el $36.84 \%$ de las empresa, seguido de un $34.74 \%$ que se ubican en un nivel medio y finalmente el $28.42 \%$ están comprendidos en un nivel alto.

Tabla 5

Nivel promedio de la rotación de inventarios de las empresas del sector industrial que cotizan en la BVL periodos 2010 al 2014

\begin{tabular}{lll}
\hline Nivel & Frecuencia & Porcentaje \\
\hline Bajo & 70 & 36.84 \\
Medio & 66 & 34.74 \\
Alto & 54 & 28.42 \\
Total & 190 & 100.00 \\
\hline
\end{tabular}

En la tabla 6 se muestra nivel de la rentabilidad económica (ROA) de las empresas del sector industrial que cotizan en la BVL periodos 2010 al 2014. Entre los tres niveles se observa que el de mayor representación es el nivel medio, y está comprendido por el $40.00 \%$, seguido de los niveles bajo y alto los cuales están comprendidos por el $30.00 \%$ respectivamente.

Tabla 6

Nivel promedio de la rentabilidad económica (ROA) de las empresas del sector industrial que cotizan en la BVL periodos 2010 al 2014

\begin{tabular}{lll}
\hline Nivel & Frecuencia & Porcentaje \\
\hline Bajo & 57 & 30.00 \\
Medio & 76 & 40.00 \\
Alto & 57 & 30.00 \\
Total & 190 & 100.00 \\
\hline
\end{tabular}

En la tabla 7 se muestra nivel de la rentabilidad financiera (ROE) de las empresas del sector industrial que cotizan en la BVL periodos 2010 al 2014. Entre los tres niveles se observa que el de mayor representación es el nivel medio, el cual está comprendido por el $40.00 \%$ de las empresas, seguido de los niveles bajo y alto los cuales están comprendidos por el $30.00 \%$ respectivamente.

\section{Tabla 7}

Nivel promedio de la rentabilidad financiera (ROE) de las empresas del sector industrial que cotizan en la BVL periodos 2010 al 2014

\begin{tabular}{lll}
\hline Nivel & Frecuencia & Porcentaje \\
\hline Bajo & 57 & 30.00 \\
Medio & 76 & 40.00 \\
Alto & 57 & 30.00 \\
Total & 190 & 100.00 \\
\hline
\end{tabular}


En la tabla 8 se presenta la correlación entre los indicadores de gestión de actividad y la rentabilidad económica de las empresas del sector industrial que cotizan en BVL 2010 al 2014. Mediante el análisis estadístico R de Pearson se obtuvo que los indicadores rotación de cuentas por cobrar (veces), periodo promedio de cobranza (días) y rotación de inventarios son altamente significativos, por lo tanto, se rechaza la hipótesis nula y se acepta la hipótesis alterna, es decir que los indicadores rotación de cuentas por cobrar (veces), periodo promedio de cobranza (días) y rotación de inventarios se relacionan favorablemente con la rentabilidad económica. Por otro lado mediante el análisis estadístico R de Pearson se obtuvo que los indicadores rotación de cuentas por pagar (veces) y la rotación de cuentas de deuda (días) no tiene una relación significativa con la rentabilidad económica, por lo tanto, se acepta la hipótesis nula y se rechaza la hipótesis alterna, es decir que los indicadores rotación de cuentas por pagar (veces) y la rotación de cuentas de deuda (días) no se relacionan favorablemente con la rentabilidad económica.

Tabla 8

Prueba de $R$ de Pearson para la relación entre los indicadores de gestión de actividad y rentabilidad económica (ROA) de las empresas sector industrial BVL periodos 2010 al 2014

\begin{tabular}{llll} 
& \multicolumn{2}{l}{ Rentabilidad Económica } & \\
\cline { 2 - 4 } & R de Pearson & P valor & $\mathrm{N}$ \\
\hline Rotación de las Cuentas por Cobrar (Veces) &, $149^{*}$ & .040 & 190 \\
Periodo Promedio de Cobranza (Días) &,$- 319^{* *}$ & .000 & 190 \\
Rotación de Cuentas por Pagar (Veces) & -.020 & .789 & 190 \\
Rotación de Cuentas de Deuda (Días) & .001 & .994 & 190 \\
Rotación de Inventarios (Veces) &, $227^{* *}$ & .002 & 190 \\
\hline
\end{tabular}

En la tabla 9 se presenta la correlación entre los indicadores de gestión de actividad y la rentabilidad financiera de las empresas del sector industrial que cotizan en BVL 2010 al 2014. Mediante el análisis estadístico R de Pearson se obtuvo que los indicadores periodo promedio de cobranza (días), rotación de inventarios y rotación de cuentas por cobrar (veces), son altamente significativos, por lo tanto, se rechaza la hipótesis nula y se acepta la hipótesis alterna, es decir que los indicadores periodo promedio de cobranza (días), rotación de inventarios y rotación de cuentas por cobrar (veces) se relacionan favorablemente con la rentabilidad financiera. Por otro lado, mediante el análisis estadístico R de Pearson se obtuvo que los indicadores rotación de cuentas por pagar (veces) y la rotación de cuentas de deuda (días) no tiene una relación significativa con la rentabilidad económica, por lo tanto, se acepta la hipótesis nula y se rechaza la hipótesis alterna, es decir que los indicadores rotación de cuentas por pagar (veces) y la rotación de cuentas de deuda (días) no se relacionan favorablemente con la rentabilidad económica.

\section{Tabla 9}

Prueba de R de Pearson para la relación entre los indicadores de gestión de actividad y rentabilidad financiera (ROE) de las empresas sector industrial BVL periodos 2010 al 2014 


\begin{tabular}{llll} 
& R de Pearson & P valor & N \\
\hline Rotación de las Cuentas por Cobrar (Veces) & .130 & .074 & 190 \\
Periodo Promedio de Cobranza (Días) &,$- 305^{* *}$ & .000 & 190 \\
Rotación de Cuentas por Pagar (Veces) & -.051 & .487 & 190 \\
Rotación de Cuentas de Deuda (Días) & .057 & .432 & 190 \\
Rotación de Inventarios (Veces) &, $226^{* *}$ & .002 & 190 \\
\hline
\end{tabular}

\section{Discusión}

El propósito de la presente investigación es la de confirmar la relación entre la gestión de actividad y la rentabilidad mediante el análisis de las empresas del sector industrial que cotizan en la BVL. Se encontró que no todas las empresas industriales informaron sus estados financieros a la Bolsa de Valores, por ello se decidió retirar a las empresas que no tenían la información requerida para este estudio. El estudio se limita a los 5 años es decir 2010 - 2014. El estudio fue basado en una data secundaria obtenida la Bolsa de Valores de Lima en la dirección web, www.bvl.com.pe. Por lo tanto, la calidad del estudio depende netamente de la precisión, fiabilidad, y disponibilidad de estos datos.

El propósito de la presente investigación es la de determinar la relación entre la liquidez y rentabilidad mediante la correlación de sus indicadores en las empresas industriales que cotizan en la Bolsa de Valores entre los periodos 2010 al 2014

No se realizó la prueba de normalidad a pesar de que los datos recogidos corresponden a 190 datos recogidos durante 5 años, en 38 empresas del sector Industrial de la Bolsa de Valores de Lima, ya que, por la cantidad de datos, se estima que mantienen una distribución normal, por lo tanto, se usó el estadístico R de Pearson para las correlaciones. No se realizó prueba piloto ya que los datos son únicos y no tienen unos segmentos similares donde poder validarlos. En la investigación los resultados giran alrededor del estudio que establece una relación entre la gestión de actividad favorablemente con la rentabilidad de las empresas del sector industrial que cotizan en la BVL.

Esto coincide con Zugarramurdi \& Parín (1999) quien asevera que los factores del capital de trabajo como el préstamo, los ingresos de efectivos y los gastos está relacionada con la rentabilidad que obtendrá una empresa industrial. De acuerdo con Pachas (1999) determina que una adecuada gestión de capital de trabajo no debería afectar la rentabilidad de la empresa. Asimismo Woll (2003) indica que la clave para mantener la rentabilidad es planificar con anticipación la gestión de las actividades y tener un flujo de caja controlado. De igual manera Castellano (2008) hace hincapié en el análisis de la gestión del capital de trabajo y la aplicación de los índices ya que son determinantes en la rentabilidad. Para Saucedo \& Oyola (2014) la gestión de los componentes del capital de trabajo se refleja en la rentabilidad esperada. De acuerdo a Soria (2013) quien cita a Cumpa y Miranda (2007) menciona que en los resultados de su investigación obtuvieron que la baja 
rentabilidad es por falta de capacitación de los microempresarios en los fundamentos de la gestión del capital de trabajo. Al igual que Rodriguez (2013) los resultados obtenidos demuestran que las decisiones financieras tomadas por la gerencia han permitido administrar adecuadamente el capital de trabajo de la empresa Consorcio Roga S.A.C por lo cual ha influido en la rentabilidad.

En cuanto a la gestión del ciclo operativo o gestión cuentas por pagar y por cobrar al igual que Horngren et al. (2000) encontramos que la gestión del ciclo operativo es determinante en la obtención de utilidades dándole mayor rentabilidad a la empresa. Coincidiendo con Guevara et al. (2010) se determina que una gestión de cuentas por pagar con mayor días de pago significará una mayor liquidez en la compañía, por ende una mayor rentabilidad y si se tiene una gestión de cuentas por cobrar en menores días la rentabilidad puede ser aún mayor. Tal como lo menciona Gonzales (2013) encontramos que el ciclo operativo es de vital para la rentabilidad de la empresa, ya que representa los recursos que ésta necesita para operar normalmente. Se demostró que el periodo promedio de cobranza esta significativamente relacionada con la Rentabilidad Económica (-,319) con un p valor igual a 0.000, al igual que Raheman \& Nasr (2007) que tuvieron como resultado del análisis de correlación muestra un coeficiente negativo - 0,165, con un valor p de $(0,000)$ de igual manera con Akgün \& Şamiloğlu (2016) existe una relación negativa y significativa entre las Cuentas por Cobrar y el ROA con un coeficiente negativo -,047, con un valor p de $(0,000)$, que a su vez coincide con Singhania et al. (2014) con un coeficiente negativo - 0.228 con un valor p de $(0,000)$, lo que significa que si el periodo promedio de cobranza aumenta, tendrá un impacto negativo en la rentabilidad y disminuirá.

Se descubrió además que las cuentas por pagar no se encuentran relacionada con la Rentabilidad Económica (.001) con un p valor igual a .994 muy diferente a los resultados de Raheman \& Nasr (2007) quienes encontraron en su correlación entre las cuentas por pagar en días donde el coeficiente es negativo y altamente significativo de -0,158 y el valor es (0,000), de igual manera Akgün \& Şamiloğlu (2016) un coeficiente negativo -,040, con un valor p de (,001). De acuerdo con Singhania et al. (2014) el coeficiente es negativo y altamente significativo de -0.170 y el valor es $(0,000)$.

Lo que significa que en nuestro país las empresas esperan menos tiempo para pagar sus cuentas a diferencia de las empresas paquistaníes que esperan demasiado tiempo para pagar sus cuentas decisión que les juega en contra porque la aceleración de los pagos a los proveedores podría aumentar la rentabilidad cuando reciben un descuento considerable por el pronto pago.

Se demostró que la rotación de inventario esta positiva y significativamente relacionada con la Rentabilidad Económica (0.227) con un p valor igual a 0.002 (p valor > 0.01), que permitió aceptar la hipótesis nula y rechazar la hipótesis alterna a diferencia de Raheman \& Nasr (2007) donde el coeficiente de correlación es 0,214 y el valor es (0,000), igualmente con Akgün \& Şamiloğlu (2016) quienes obtuvieron en su investigación que existe una relación negativa entre la rotación de inventarios y el ROA con un coeficiente de correlación es -,006 y el valor es $(, 615)$ con un resultado poco significativo, esto indica que si la empresa toma más tiempo en la venta de inventario, afectará negativamente a su rentabilidad, coincidiendo con Singhania et al. (2014) con un coeficiente de correlación negativo -0.067 y el valor es $(0,085)$. Igualmente Peralta \& Perez (2013) 
además que para Bianchi (2012) cuanto mayor es el índice, cuantas más son las vueltas al año, mejor es la gestión del inventario y su impacto en la rentabilidad. Al igual que Gonzales (2014) descubrimos que una disminución de las cuentas por pagar significaría una rentabilidad económica menor. Arcos \& Benavides (2008) mencionan que en un estudio de Laziridis y Tryofinidis (2006) encontraron que la relación entre estas medidas de rentabilidad y las cuentas por pagar es positiva y que entre las medidas de rentabilidad y los días de cuentas por cobrar son también negativas.

\section{Conclusiones}

Para el primer objetivo, se concluye que no existe una relación entre la gestión de cuentas por pagar y la rentabilidad económica de las empresas industriales que cotizan en la BVL entre los periodos 2010 al 2014. Por lo tanto, se acepta la hipótesis nula, encontrando que un nivel bajo de gestión de cuentas por pagar y rentabilidad financiera no se relaciona.

Para el segundo objetivo, se concluye que no existe una relación entre la gestión de cuentas por pagar y la rentabilidad financiera de las empresas industriales que cotizan en la BVL entre los periodos 2010 al 2014. Por lo tanto, se acepta la hipótesis nula, encontrando que un nivel bajo de gestión de cuentas por pagar y rentabilidad financiera no se relaciona.

Para el tercer objetivo, se concluye que existe una relación entre la gestión de cuentas por cobrar y la rentabilidad económica de las empresas industriales que cotizan en la BVL entre los periodos 2010 al 2014. Por lo tanto, se acepta la hipótesis alterna, el tipo de relación que presenta estos indicadores es directa puesto que el índice de correlación Rotación de las Cuentas por Cobrar (Veces) de Pearson ,149 se encuentra en el nivel .040 y el Periodo Promedio de Cobranza (Días) de Pearson -,319 se encuentra en el nivel .000 siendo ésta significativa. Para el cuarto objetivo, se concluye que existe una relación entre la gestión de cuentas por cobrar y la rentabilidad financiera de las empresas industriales que cotizan en la BVL entre los periodos 2010 al 2014. Por lo tanto, se acepta la hipótesis alterna, el tipo de relación que presenta estos indicadores es directa puesto que el índice de correlación Rotación de las Cuentas por Cobrar (Veces) de Pearson ,130 se encuentra en el nivel .074 y el Periodo Promedio de Cobranza (Días) de Pearson -,305 se encuentra en el nivel .000 siendo ésta significativa. Para el quinto objetivo, se concluye que existe una relación entre la gestión de cuentas por cobrar y la rentabilidad económica de las empresas industriales que cotizan en la BVL entre los periodos 2010 al 2014. Por lo tanto, se acepta la hipótesis alterna, el tipo de relación que presenta estos indicadores es directa puesto que el índice de correlación Rotación de las Rotación de Inventarios (Veces) de Pearson ,227 se encuentra en el nivel .002 y siendo ésta significativa. Para el sexto objetivo, se concluye que existe una relación entre la gestión de cuentas por cobrar y la rentabilidad financiera de las empresas industriales que cotizan en la BVL entre los periodos 2010 al 2014. Por lo tanto, se acepta la hipótesis alterna, el tipo de relación que presenta estos indicadores es directa puesto que el índice de correlación Rotación de las Rotación de Inventarios (Veces) de Pearson ,226 se encuentra en el nivel .002 y siendo ésta significativa. Por tanto, se puede afirmar que existe correlación entre los indicadores de gestión de actividad y la rentabilidad económica de las empresas del sector industrial que cotizan en BVL 2010 al 2014. Mediante el análisis estadístico R de 
Pearson se obtuvo que los indicadores rotación de cuentas por cobrar (veces), periodo promedio de cobranza (días) y rotación de inventarios son altamente significativos, por lo tanto, se rechaza la hipótesis nula y se acepta la hipótesis alterna, es decir que los indicadores rotación de cuentas por cobrar (veces), periodo promedio de cobranza (días) y rotación de inventarios se relacionan favorablemente con la rentabilidad económica.

\section{Referencias}

Aching, C., \& Aching, J. (2006). Ratios financieros y matemáticas de la mercadotecnia (Vol. 1). Recuperado de http://doi.org/10.1017/CBO9781107415324.004

Aguilar Pinedo, V. H. (2013). Gestión de cuentas por cobrar y su incidencia en la liquidez de la empresa contratista Corporación Petrolera S.A.C.- año 2012. Universidad San Martín de Porres.

Akram, M., Jamil, S., Ali, N., Ali, B., \& Khan, W. (2016). Impact of Working Capital Management on Market Return : A Comparative Study of Pakistan and Indian Chemical Sector, 5(10), 10-25.

Alarcón Reyes, Gigi Thalía; Rosales Larrea, I. M. (2015). Evaluacion de la Gestión Económia-Financiera y su implicancia en la rentabilidad de la empresa "panificación arte distribuidores SAC" de la ciudad de Chiclayo. Universidad Católica Santo Toribio de Mogrovejo.

Alarcón, G., \& Rosales, I. (2014). Evaluacion de la Gestión Económica - Financiera y su implicancia en la rentabilidad de la empresa "Panificación arte distribuidores SAC" de la ciudad de Chiclayo durante el periodo 2014.

Arcos, M., \& Benavides, J. (2008). Efecto del Ciclo de Efectivo sobre la Rentabilidad de las Firmas Comlombianas, 21(36), 167-182. Retrieved from http://revistas.javeriana.edu.co/index.php/cuadernos_admon/article/viewFile/3943/2913

Barajas, A. (2008). Finanzas para no financistas. Retrieved from http://www.bibliociencias.cu/gsdl/collect/libros/index/assoc/HASH6cb4.dir/doc.pdf

Bernal, M. (2004). Contabilidad, sistema y gerencia. Retrieved from https://books.google.com.pe/books?id=8w6XMU_drbMC\&printsec=frontcover\&hl=es\&source =gbs_ge_summary_r\&cad $=0 \# \mathrm{v}=$ onepage $\& \mathrm{q} \& \mathrm{f}=\mathrm{false}$

Carrasco, D. (2014). Análisis comparativo de rentabilidad, eficiencia y concentración entre bancos y microfinancieras durante el periodo 2005-2011. Universidad Católica Santo Toribio de Mogrovejo.

Castellano, Y. (2008). Análisis del capital de trabajo desde la perspectiva de la Rentabilidad - Riesgo en las empresas constructoras del Municipio Valera.

Choy, C. (2008). La importancia de una efectiva administración del efectivo a corto plazo. Retrieved from http://glifos.unis.edu.gt/digital/tesis/2008/22747.pdf 
Dávila, C. (2015). Diagnostico de liquidez en la empresa Heladerías COFRUNAT CÍA LTDA. y propuesta de un plan de acción utilizando el modelo de gestión centuria. Retrieved from http://repositorio.puce.edu.ec/bitstream/handle/22000/11020/TESIS MODELO CENTURIA.pdf? sequence $=1 \&$ isAllowed $=\mathrm{y}$

De Jaime, J. (2003). Análisis económico-financiero de las decisiones de gestión empresarial. (Vol. 1). ESIC. http://doi.org/10.1017/CBO9781107415324.004

De LaHoz Suárez, B., Ferrer, M. A., \& De La Hoz Suárez, A. (2008). Indicadores De Rentabilidad: Herramientas Para La Toma De Decisiones Financieras En Hoteles. Revista de Ciencias Sociales, XIV, 88-109.

De Mariana, J. (1854). Obras del padre Juan de Mariana (Vol. 2). Rivadeneyra, Manuel. http://doi.org/10.1017/CBO9781107415324.004

Desposorio, J., \& Espinoza, M. (2011). Propuesta e implementación de la gestión del proceso logistico par la mejora de los resultados del capital de trabajo de la Empresa distribuciones Uriol EIRL 2011.

Ehrhardt, M., \& Brigham, E. (2007). Finanzas Corporativas. Retrieved from https://books.google.com.pe/books?id=bLOKcYHCOdsC\&dq=ciclo+de+efectivo\&hl=es\&source=gbs_ n avlinks_s

Espinoza, D., \& Selpa, A. (2004). La gestión del capital de trabajo como proceso de la gestión financiera operativa. Ajoica Journal, 9. Retrieved from https://dialnet.unirioja.es/servlet/articulo?codigo=3113464 Francés, A. (2006). Estrategia y planes para la empresa: con el cuadro de mando integral. (P. Educación, Ed.). Retrieved from https://books.google.com.pe/books?id=yAmLGVr8BkC\&dq=ciclo+de+efectivo\&hl=es\&source=gbs_navlinks_s

Gamboa, A. (2015). Aplicación de la auditoria de gestión como herramienta de control y evaluación para mejorar la rentabilidad en las empresas comercializadoras d vehiculos de la provincia de Trujillo.

García Restrepo, L. Y. (2013). Liquidez y Rentabilidad como factor determinante en el éxito de las empresa. Journal of Chemical Information and Modeling, 15.

García, F. (2015). El ciclo de conversioón del efectivo y su importancia en la liquidez de la empresa Fabiola S.A. Retrieved from http://repositorio.utmachala.edu.ec/bitstream/48000/3275/1/ECUACE-2015-CACD00109.pdf

Gitman, L. (2003). Principios de administración financiera. Retrieved from https://books.google.com.pe/books?id=KS_04zILe2gC\&printsec=frontcover\&hl=es\&source=gbs_ge_s ummary_r\&cad $=0 \# \mathrm{v}=$ onepage $\& \mathrm{q} \& \mathrm{f}=$ false

Gonzales, E. (2013). La administración del capital de trabajo en la gestión de las empresas distribuidoras de medicinas de Lima Metropolitana. 
Guevara, I., Prudencio, P., \& Quintanilla, D. (2010). Analisis de los flujos de efectivo para evaluar la liquidez

y el cumplimiento de obligaciones de corto y largo plazo en la empresa que comercializa pinturas, barnices, productos de revestimiento y similares. Retrieved from http://ri.ues.edu.sv/347/1/10136955.pdf

Horngren, C., Sundem, G., \& Elliott, J. (2000). Introducción a la contabilidad financiera. (Pearson Educación, Ed.). http://doi.org/10.1017/CBO9781107415324.004

Jamalinesari, S., \& Soheili, H. (2015). The Relationship between the Efficiency of Working Capital Management Companies and Corporate Rule in Tehran Stock Exchange. Procedia - Social and Behavioral Sciences, 205(May), 499-504. http://doi.org/10.1016/j.sbspro.2015.09.052

Lee j, Krajeswski; Larry P, R. (2000). Administración de operaciones: estratégia y análisis.

Lizarraga, V., K. (2010). Gestión de las cuentas por cobrar en la administración del capital de trabajo de las empresas de fabricación de plástico del distrito de ate, 174.

Locke, J. (2010). Segundo tratado sobre el gobierno civil. (Tecnos, Ed.) (Segunda ed). España. Retrieved from https://dairoorozco.files.wordpress.com/2013/01/locke-segundo-tratado-sobre-el-gobiernocivil.pdf

Mejía, V. (2014). Control de la morosidad como estrategia para mejorar el nivel de rentabilidad en la financiera Crediscotia de la ciudad de Huaraz periodo 2012.

Meza, V. I. (2013). La gestión estratégica de costos en la rentabilidad en las empresas de ensamblaje e instalación de ascensores en Lima Metropolitana, año 2012.

Moreno, N. (2004). Ciclo De Conversión De Efectivo En Las Grandes Empresas De Comercio Al Detal En Colombia (1998-2002). Estudios Gerenciales, 20(92), 129-138. Retrieved from http://www.scielo.org.co/scielo.php?script=sci_arttext\&pid=S0123$59232004000300005 \& \operatorname{lng}=\mathrm{en} \& n r m=\mathrm{iso} \& \operatorname{lng}=\mathrm{es}$

Morillo, M. (2001). Rentabilidad Financiera y Reducción de Costos. Actualidad Contable Faces, 4(4), 35-48.

Muro Fuentes, L. C. (2014). Diagnóstico Económico Financiero y su implicancia en la proyección de Estados Financieros al año 2015 de la empresa Agroindustrial de Lambayeque-2013. Universidad Católica Santo Toribio de Mogrovejo.

Nevado, D., López, V., Pérez-Carballo, J., \& Zaratiegui, J. (2007). Cómo gestionar el binomio rentabilidadproductividad: Función económico- financiera, la gestión de procesos, la gestión de la calidad y el cuadro de mando integral. Journal of Chemical Information and Modeling, 296. http://doi.org/10.1017/CBO9781107415324.004

Ordaz-díaz, J. L. (2008). Rentabilidad económica de la educación en México: comparación entre el sector urbanoy el rural.

Otal, F., Sara, H., Serrano, G., \& Serrano, R. (2007). Simulación financiera con delta Simul-e (Vol. 53). 
Pachas, P. (1999). Rentabilidad del capital de trabajo copetitividad, 6.

Pandey, N., \& Sabamaithily, S. (2016). Working Capital Management on Profiability: Cement Industry in India. SCMS Journal of Indian Management, 02(02), 81-96.

Paucar, E. (2015). Evaluación del ciclo operativo de producción agricola y su incidencia en la estructura economica de las empresas agroindustriales del valle del Virú.

Peralta, P., \& Perez, X. (2013). Analisis de la rotación de inventarios versus la rotación de cartera y su impacto en el flujo de efectivo aplicado a la compañía industrias Guapán S.A periodo 2011 - 2012. Retrieved from http://dspace.ucuenca.edu.ec/bitstream/123456789/3555/1/TESIS.pdf

Pérez, C. (2014). La calidad del servicio al cliente y su influencia en los resultados economicos y financieros de la empresa Restaurante Campestre S.A.C - Chiclayo periodo Enero a Setiembre 2011 y 2012. Retrieved from www.conocimientosweb.net/descargas/article1087.html

Pérez, K., Socarrás, F., \& Labrada, E. (2014). Procedimientos Metodológicos Para La Administración Del Efectivo. Observatorio de La Economía Latinoamericana, 194, 1-21. Retrieved from http://www.eumed.net/cursecon/ecolat/cu/2014/administracion-efectivo.html

Pérez-Carballo, J. (2013). Control de gestión empresarial. Retrieved from https://books.google.com.pe/books?id=06ptFLzbjjMC\&dq=rentabilidad+economica\&source=g bs_navlinks_s

Perez-Carballo, J., \& Perez-Carballo, J. F. (1998). Compitiendo por crear valor. ESIC. http://doi.org/10.1017/CBO9781107415324.004

Phala, L. (2015). Gestión de costos de servicios y rentabilidad de hotelería e inversiones Latino S.A - Hotel Tierra Mística Qalasaya de la ciudad de Puno, periodos 2009 - 2010.

Quintero, J. (2009). Planificación financiera. Retrieved from http://www.eumed.net/ce/2009b/jcqp2.zip.

Quiroz, C. (2011). Planteamiento estrategico par aumentar el saldo de colocación del área de credito credijoya de la caja municipal de ahorro y credito de Trujillo agencia avenida España para el año 2011 .

Raheman, A., \& Nasr, M. (2007). Working Capital Management And Profitability - Case Of Pakistani Firms. International Review of Business Research Papers, 3(1), 279-300.

Rivera, I., \& Morúa, J. (2011). Reconstrucción del enfoque del aumento y generación del efectivo para la Pyme de manufactura. Contaduría Y Administración, (234), 131-150.

Rodriguez Castro, D. A. (2013). Administración Del Capital De Trabajo Y Su Influencia En La Rentabilidad De La Empresa Consorcio Roga S.A.C. Trujillo 2011-2012. Universidad Privada Antenor Orrego. 
Sanchez, M. (2013). Administración eficiente del efectivo, sostenibilidad asegurada en el largo plazo.

Retrieved from

http://repository.unimilitar.edu.co/bitstream/10654/9836/1/SanchezAlbaMariaConstanza2013.pdf

Saucedo Carrero, Duvert Luis; Oyola Alvites, E. L. (2014). La administracion del Capital de Trabajo y su influencia en la Rentabilidad del club deportivo ABC S.A-Chiclayo, en los periodos 2012 - 2013. Universidad Católica Santo Toribio de Mogrovejo.

Saucedo, D., \& Oyola, E. (2014). La administración del capital de trabajo y su influencia en la rentabilidad del club deportivo ABC S.A - Chiclayo, en los periodos 2012-2013. Universidad Catolica Santo Toribio de Mogrovejo.

Singhania, M., Sharma, N., \& Yagnesh, J. (2014). Working capital management and profitability: evidence from Indian manufacturing companies. Decision, 41(3), 313-326. http://doi.org/10.1007/s40622-0140043-3

Smith, A. (1794). La riqueza de las naciones. (O. de V. e H. de Santander, Ed.). Valladolid. Retrieved from http://www.uv.es/ mpuchade/MDH/02_Smith.pdf

Soles, Lady. (2015). Diseño e implementación de un sistema de control interno y su influencia en la rentabilidad de la empresa Negocios e Inversiones integrales del Perú EIRL., e el distrito de Trujillo, periodo 2012.

Tanaka, G. (2001). Análisis de estados financieros para la toma de decisiones. (F. E. PUCP, Ed.). Retrieved from

https://books.google.com.pe/books?id=LH4fWKr2Cs4C\&pg=PA218\&dq=ciclo+de+efectivo\&hl= es\&sa=X\&ved=0ahUKEwiIrKzEt5LOAhWFox4KHQ3VBIUQ6AEIITAB\#v=onepage \&q=ciclo de efectivo\&f=false

Tong, J. (2006). Finanzas empresariales: La decisión de inversión.

http://doi.org/10.1017/CBO9781107415324.004

Torres, I. (2012). Mejoramiento de la gestión del capital de trabajo y del ciclo de efectivo, en la empresa NR Productos industriales y agricolas S.A., ubicada en la provincia de Pichincha, Quito - Ecuador. PONTIFICIA UNIVERSIDAD CATOLICA DEL ECUADOR. Retrieved from http://repositorio.puce.edu.ec/bitstream/handle/22000/5146/T-PUCE5372.pdf? sequence $=1 \&$ is Allowed $=\mathrm{y}$

Van Horne, J., \& Wachowicz, J. (2002). Fundamentos de la administración financiera. 
Vargas, R. (2011). Evaluación del ciclo de efectivo de CVG Aluminios nacionales y su efecto en la liquidez de la empresa. Retrieved from http://bb9.ulacit.ac.cr/tesinas/publicaciones/032485.pdf

Zelada, H. (2014). Propuestas de implementación de un sistema de costos por procesos e al cría intensiva de ganado porcino para mejorar la rentabilidad economica y financiera de la empresa Granja San Luis E.I.R.L Guadalupe - La Libertad, año 2013. 